\title{
Flow characteristics by particle image velocimetry in liquefied natural gas vaporizer model with several baffles
}

\author{
H. S. Chung ${ }^{1}$, S. M. Sayeed-Bin-Asad ${ }^{2}$, Berkah Fajar ${ }^{3}$, Y. H. Shin ${ }^{2}$, H. M. Jeong ${ }^{1}$ \\ 1. Department of Mechanical and Precision Engineering, Institute of Marine Industry, \\ 445 Inpyeong Dong, Tongyeong, Gyeongsangnamdo, 650-160, Korea; \\ 2. Department of Mechanical and Precision Engineering, Gyeongsang National University, \\ 445 Inpyeong Dong, Tongyeong, Gyeongsangnamdo, 650-160, Korea; \\ 3. Department of Mechanical Engineering, University of Diponegoro, Semarang, Indonesia
}

(C) Central South University Press and Springer-Verlag Berlin Heidelberg 2011

\begin{abstract}
Shell-and-tube vaporizers are the most commonly used and dominated types of vaporizers in liquefied natural gas (LNG) realm. Due to efficient performance, shell-side flow in this type of vaporizers has received considerable attention and has been investigated extensively. However, the detailed flow structure in the shell needs to be determined for reliable and effective design. Therefore, the objective of this study was to clarify the flow structure in shell by particle image velocimetry (PIV). Experiments were conducted using two types of model; $15 \%$ baffle cut having inlet and outlet positions in the direction of $90^{\circ}$ to the cut and $30 \%$ baffle cut having inlet and outlet positions in the direction of $180^{\circ}$ to the cut. Each test section is $169 \mathrm{~mm}$ in inner diameter and $344.6 \mathrm{~mm}$ in length. The flow features were characterized in different baffle cuts with regards to the velocity vector field and velocity distribution. The results show that the flow characteristics of $15 \%$ baffle cut type vaporizer are comparable to those of $30 \%$ baffle cut type vaporizer.
\end{abstract}

Key words: particle image velocimetry; liquefied natural gas; vaporizer; vortex; turbulence

\section{Introduction}

Liquefied natural gas (LNG) is a simply natural gas in its liquid form. Natural gas is converted to LNG by cooling it to $-162{ }^{\circ} \mathrm{C}$ at atmospheric pressure. This process reduces its volume by a factor of more than 600 , which allows natural gas to be transported efficiently between continents by specially designed ocean vessels. Thus, LNG technology makes natural gas available throughout the world. Nowadays, world demand for LNG is surprisingly increasing.

Once LNG reaches the desired destination, it is unloaded from ships then fed into a regasification plant to return LNG to a gaseous state. The LNG is warmed by passing through pipes heated by direct-fired heaters, seawater or through pipes that are in heated water. These devices are known as 'vaporizers'. A vaporizer in fact is a vaporizer [1], but one of the main obstacles that occur in a vaporizer is frost formation due to the cryogenic condition of LNG, which reduces the heat transfer efficiency of the system. The vaporized gas is then regulated for pressure and enters the pipeline system as natural gas. Finally, residential and commercial consumers receive natural gas for daily use from local gas utilities or in the form of electricity.

However, in respect of effective design, prediction of flow across shell of shell and tube vaporizer is very important [2]. The problem is greatly complicated by the variations of configurations encountered in practice resulting in different flow patterns, and by the effects of flow parameters. Following this, shell-side flow in shell and tube vaporizer (STV) has received considerable attention and has been investigated. IWAKI et al [3] studied vertical cross-flow in shell and tube heat exchanger. ZDRAVKOVICH [4] reviewed previous studies concerning interstitial flow extensively. According to his literature, early investigations were concerned with flow visualization and some successful visualizations of the interstitial flow were reported. One of the first successful visualizations using hydrochloric acid and ammonia vapor was carried out by LORISCH [5]; he made boundary layers and wakes visible. PENDENNIS-WALLIS [6] also made a visualization of

Foundation item: This work was supported by the National Research Foundation of Korea Grant funded by the Korean Government (NRF-2010-013D00007) and 2010 Research Professor Fund of Gyeongsang National University, Korea

Received date: 2011-02-09; Accepted date: 2011-03-28

Corresponding author: H. M. Jeong, Professor, PhD; Tel: +82-55-640-3184; Fax: +82-55-640-3188; E-mail: hmjeong@gnu.ac.kr 
interstitial flow in water for an inline array with four rows, and noticed that wide and narrow near wakes were formed together around tubes at the same row. BRADSHAW [7] performed smoke visualization in a single row and noted that flow behind tubes of the single row was non-uniform, in spite of constant pitch and right angles in the uniform stream. ZDRAVKOVICH [8] observed a flow pattern as well as pressure distribution and suggested that such non-uniformity caused flow-induced vibration. WEAVER and AVD-RABBO [9] made a visualization of water interstitial flow for stationary tubes and vibrating tubes, which indicated that tube vibration produced wide and narrow jets that remained straight. However, these studies had a lack of quantitative evaluation and sufficient measurement, although they revealed qualitative characteristics of cross-flow.

However, in order to establish reliable design and performance criteria for tube bundle models, more detailed and accurate data are needed. With respect to the velocity distribution in a tube bundle, some experiments were conducted using laser Doppler anemometry (LDA) for specific tube arrangements. SIMONIN and BARCOUDA [10] measured the velocity for flow in tube bundles by LDA and compared the results to k-epsilon model predictions. BALABANI and YIANNESKIS [11] estimated a turbulent scale by velocity measurement with LDA, and they revealed that a staggered array generated a higher level of turbulence compared with an in-line array. Although these previous studies presented velocity characteristics for cross-flow over a tube bundle, which may be attributed to the understanding of cross-flow characteristics, the measurement points were limited in number and detailed velocity distributions or the whole flow field data have not been obtained. In these studies, there is a lack of data, particularly for the region close to the tubes. Recently, UMEDA and YANG [12] reported a lot of visualization results by using a particle tracing method and velocity measurement results at some points by LDA, although the detailed velocity data were not obtained.

Particle image velocimetry (PIV) has been lately developed for flow measurements in a wide region of flow field. It can offer many advantages for the study of fluid flow over a conventional one-point measurement, such as LDA or hot wire anemometry. PIV can measure the velocity in a whole flow field instantaneously, without bothering the structure of the complicated flow field, and it would be particularly effective for the flow in which some structures exit. Therefore, in this study, PIV was adopted to clarify the flow structure by obtaining detailed velocity data in the whole flow area of shell. And different baffle designs with different inlet flow positions were focused on.

\section{Experimental}

\subsection{Experimental apparatus}

Figure 1 shows two configurations of the test section we used; $15 \%$ and $30 \%$ baffles cuts. The test section had a circular cross-section with inner diameter of $169 \mathrm{~mm}$ and length of $344.6 \mathrm{~mm}$. The detailed feature of the model is listed in Table 1. The test vaporizer and the baffles were, respectively, made of transparent acrylic tube and acrylic sheet in order to enable the laser sheet to pass through them and reach the whole measurement area. The test section was set into the test loop, as shown in Fig.2. The test loop mainly consisted

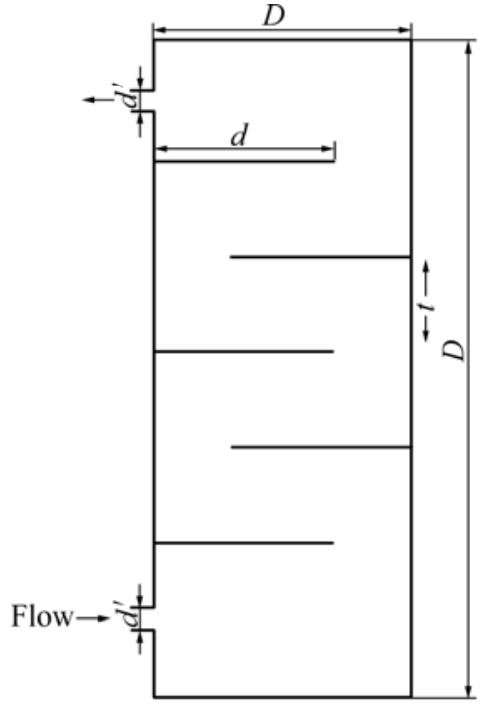

(a)

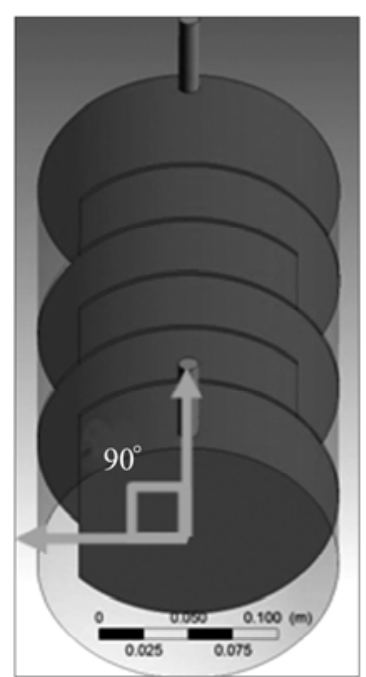

(b)

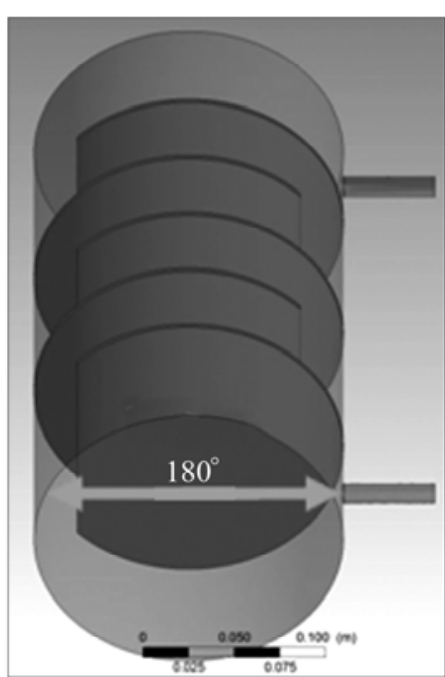

(c)

Fig.1 Test section (Dimension of shells: $D=169 \mathrm{~mm}$ and $d^{\prime}=11.4 \mathrm{~mm}, L=344.6 \mathrm{~mm}, t=50 \mathrm{~mm}$ apart, $d=143.65 \mathrm{~mm}(15 \%$ BC) and $118.3 \mathrm{~mm}(30 \% \mathrm{BC}))$ : (a) Model; (b) $15 \% \mathrm{BC}$; (c) $30 \% \mathrm{BC}$ 
Table 1 Main dimensions and features of STV model

\begin{tabular}{cc}
\hline Item & Value and description \\
\hline Inside diameter of shell/mm & 169 \\
Length of shell $/ \mathrm{mm}$ & 344.6 \\
Diameter of Baffle $/ \mathrm{mm}$ & 145.6 \\
Baffle thickness $/ \mathrm{mm}$ & 3 \\
Baffle spacing $/ \mathrm{mm}$ & 50 \\
Number of baffle & 5 \\
Type of baffle & Single segmental \\
Baffle cut (BC) & $15 \%$ or 30\% of \\
\end{tabular}

of a tank, test section, circulation pump and flow meter. The speed of the pump was controlled to set the flow rate to a fixed value and the flow rate was measured by a flow meter.

\subsection{Measurement method}

In this study, the PIV method was adopted to measure the flow in the shell in order to obtain detailed velocity data in the whole flow area. PIV with a green DPSS laser was employed and its wavelength was $532 \mathrm{~nm}$, as shown in Fig.3. The laser light was fanned into a thin longitudinal sheet which illuminated a plane on the centerline of the test section. The CCD camera was used to record the flow image data. The laser beam and the CCD camera were set in a way for measuring area in the flow field of the test section. Table 2 lists the details of experimental parameter. The image data of the flow area were acquired.

With a CCD camera and stored as separate frames for each illumination pulse, the two images acquired at short time intervals were processed by a cross-correlation method, in which the cross-correlation coefficient was evaluated in a small sub-region of images to obtain a velocity vector field.

\subsection{Experimental condition}

The experiments were carried out under atmospheric pressure and three different Reynolds numbers 9 300, 4500 and 2500.

The Reynolds number is defined as

$$
R e=\frac{\rho v_{0} d}{\mu}
$$

where $v_{0}$ is the inlet flow velocity, $d$ is the inlet diameter of the test section, $\rho$ is the density of water and $\mu$ is the viscosity of the water at $20{ }^{\circ} \mathrm{C}$. The time interval between the illuminated laser pulses was varied from $0.1 \mathrm{~ms}$ to $2.0 \mathrm{~ms}$, corresponding to the sectional averaged velocity. The experiments were conducted at room temperature, set at $20{ }^{\circ} \mathrm{C}$, with the working fluid temperature maintained between 20 and $25^{\circ} \mathrm{C}$.

\section{Experimental results}

\subsection{Velocity field and component distribution for $\boldsymbol{R} e=$ 9300}

Figure 3 shows the results of the velocity vector field obtained for the $15 \% \mathrm{BC}$ and $30 \% \mathrm{BC}$ configurations in the whole flow areas of the test sections at $v_{0}=0.82 \mathrm{~m} / \mathrm{s}$ that also allows to have the same $\operatorname{Re}(=9300)$. One thousand frames of images were recorded every $0.2 \mathrm{~ms}$ and averaged to obtain the mean velocity. Figures 3(a) and 3(b) show the velocity vectors in the shells. Both figures show flow pattern along the shells which are little different from one another due to different baffle configurations; a separated compartment layer and recirculation flow were formed in every compartment of shells. In the case of 15\% BC (Fig.3(a)), flow field was characterized by flow regions of layer and recirculation flow formed in every baffle compartment, i.e., a vortex

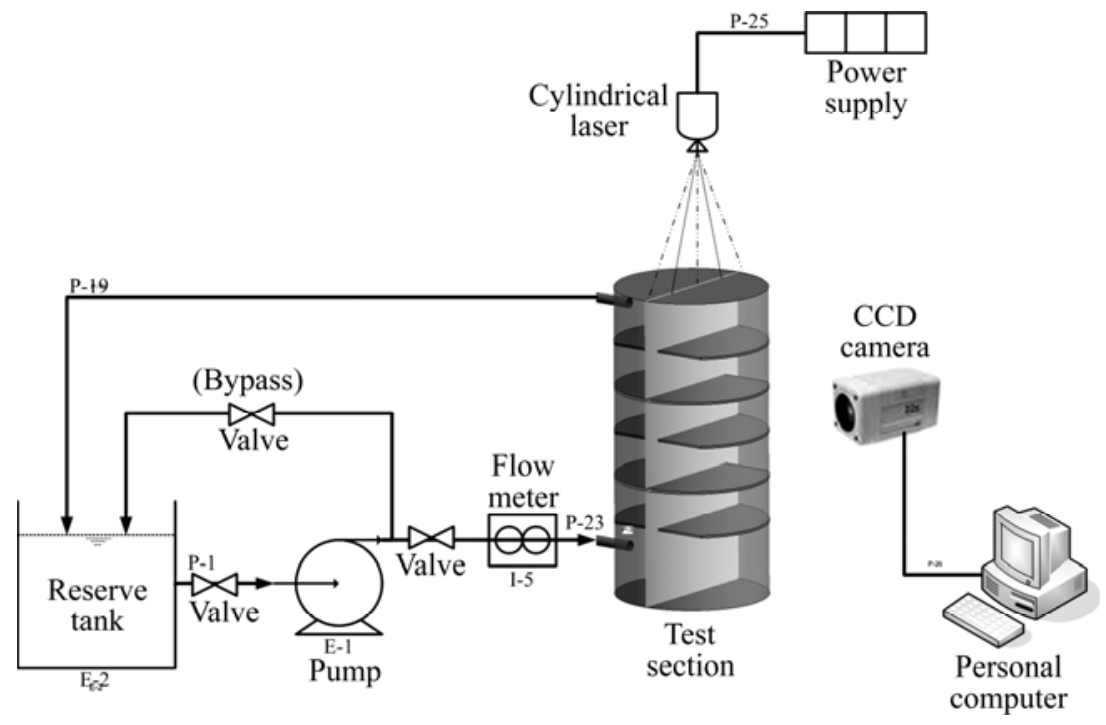

Fig.2 Schematic diagram of test loop 

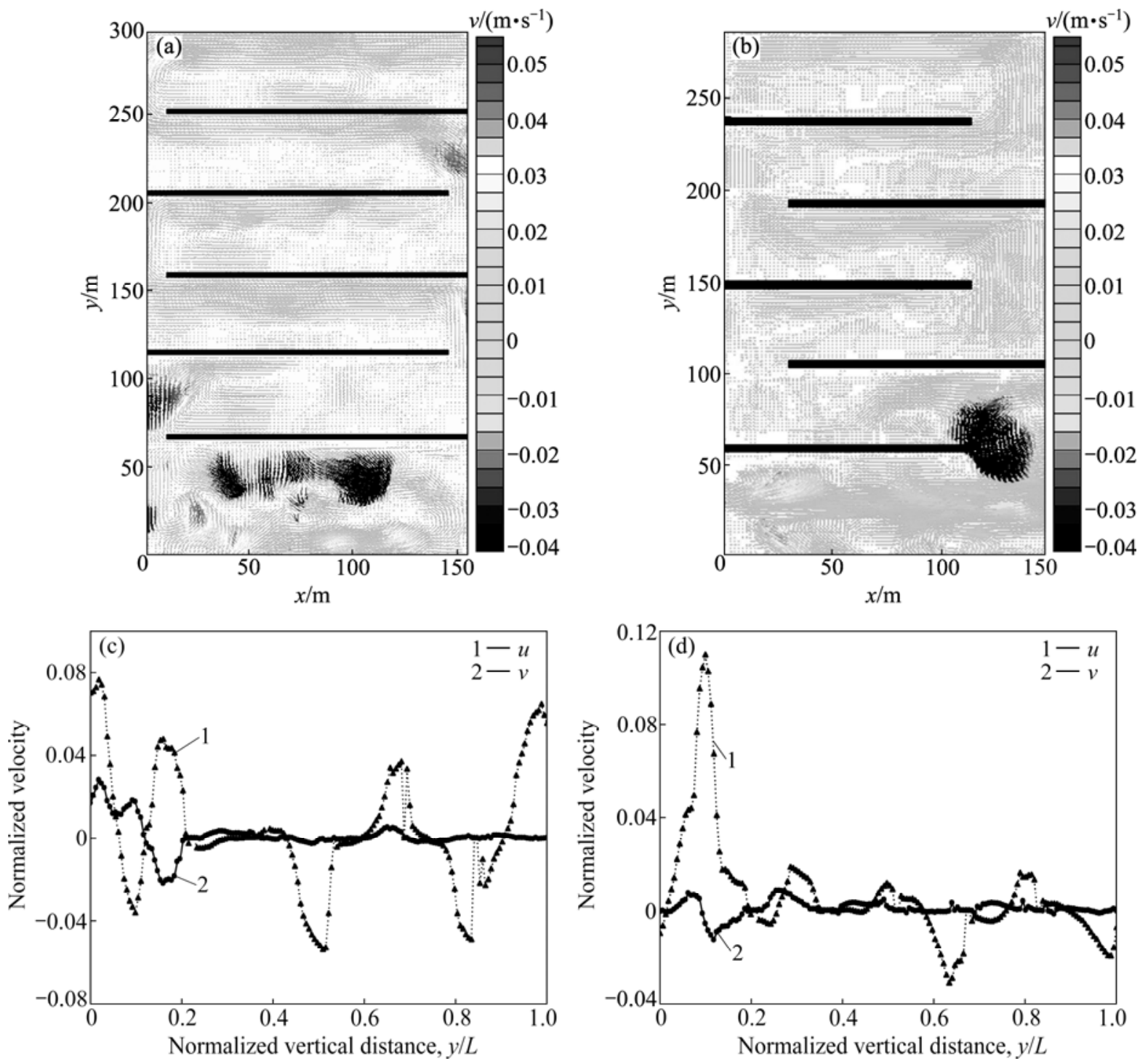

Fig.3 Velocity vector fields ((a) and (b)) and component distributions ((c) and (d)) of shell for $R e=9$ 300: (a) and (c) $15 \%$ BC; (b) and (d) $30 \% \mathrm{BC}$

Table 2 Experimental parameters

\begin{tabular}{cc}
\hline Item & Value and description \\
\hline Seeding particle & PVC (Poly Vinyl Choloride) \\
Particle size $\mu \mathrm{m}$ & 200 \\
Water fluid & Water \\
Light source & Green DPSS Laser \\
Sheet light & Cylindrical Lens \\
Image recorder & CCD Camera \\
\hline
\end{tabular}

region and a high velocity region in the main flow. The figure also shows that the pattern of the vortex generated in shell was different for each compartment.

On the other hand, Fig.3(b) shows that the flow in the $30 \%$ baffle cut configuration had a remarkably different pattern from that in the $15 \%$ baffle cut. Adjacent baffle and wall of the shell restricted the mainstream velocity, which makes flow cross throughout the entire shell. Therefore, the flow field was characterized by the flow region of every compartment. It was noticed that similar type of vortices in compartments except inlet and outlet was produced and a Karman vortex was not seen in any compartment of the entire shell. The reasons why this remarkable difference was noticed are the inlet flow condition and baffle cut. Figures 3(c) and 3(d) show the velocity distributions in the $y$ direction under the flow condition of $R e=9300$ in the inlet area for $15 \% \mathrm{BC}$ and $30 \% \mathrm{BC}$ configurations, respectively. The $x$ and $y$ components of velocity, $u$ and $v$, were respectively normalized by $v_{0}$. In the $15 \% \mathrm{BC}$ configuration, the results show that $v$ had a maximum value bottom compartment but in all other compartments, value of $v$ was noticed almost steady. The maximum value of $u$ was noticed in bottom and top compartment of the shell. Similarly, the third and top compartments were 
seen to have the minimum value of $u$ and this is due to the baffle configuration.

On the other hand, in the $30 \% \mathrm{BC}$ configuration, the results show that $v$ had a maximum fluctuation in bottom compartment but in all other compartments, the value of $v$ was noticed almost steady throughout the entire shell and this is because of the inlet flow direction. There was remarkable maximum value of $u$ in only bottom compartment of the shell. Similarly, $u$ was noticed to have the minimum value in the third and top compartments of the shell. These velocity distributions suggest that the flow mixing was more promoted in the $15 \% \mathrm{BC}$ than in the $30 \% \mathrm{BC}$ because of inlet flow direction and baffle cut.

\subsection{Velocity field and component distribution for $R e=$ 4500}

The velocity vector field and component distribution obtained for the $15 \% \mathrm{BC}$ and $30 \% \mathrm{BC}$ configurations in whole flow areas of the test sections at same $v_{0}=0.40 \mathrm{~m} / \mathrm{s}$
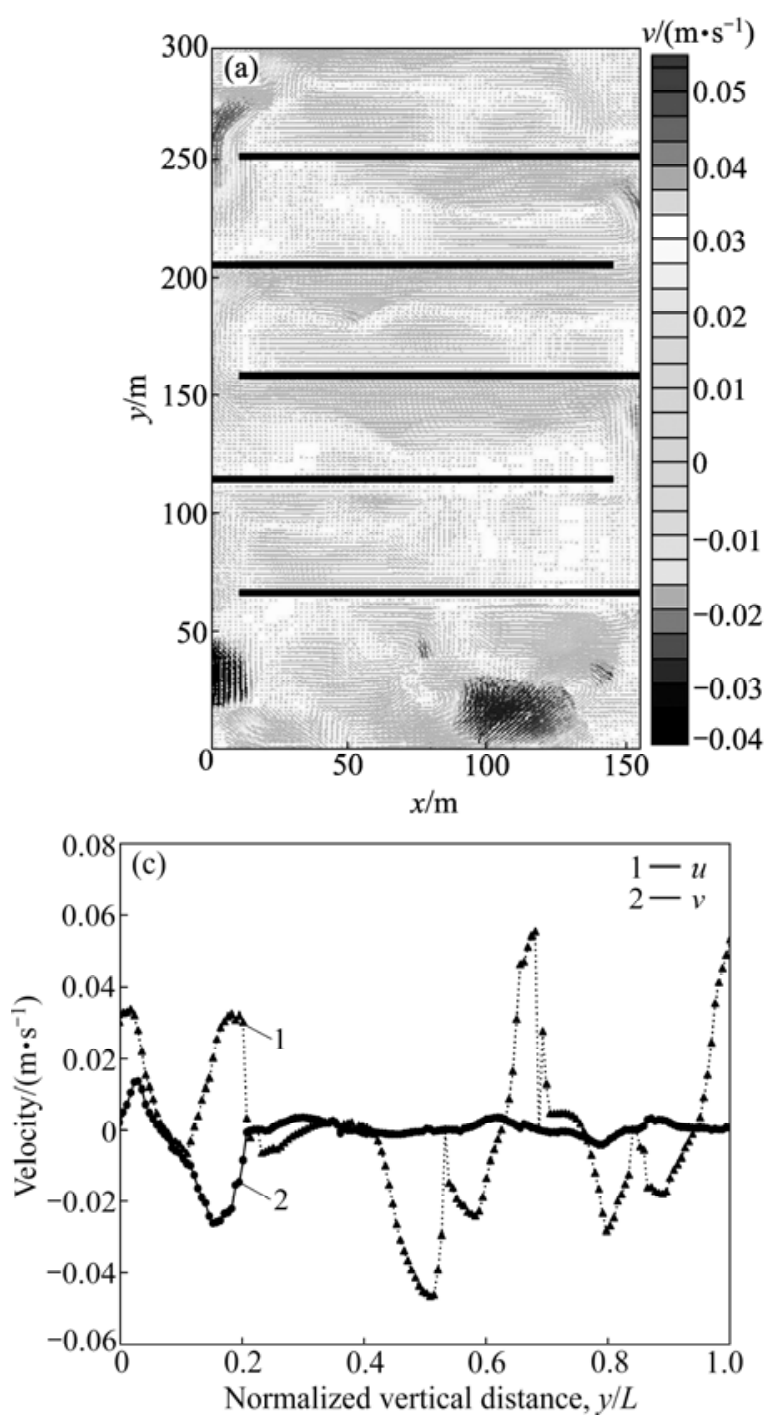

Fig.4 Velocity vector fields ((a) and (b)) and component distributions ((c) and (d)) of shell for $R e=4500$ : (a) and (c) $15 \%$ BC; (b) and (d) $30 \% \mathrm{BC}$ that also allows to have the same $\operatorname{Re}(=4500)$ which is shown in Fig.4. Figures 4(a) and 4(b) show the velocity vector in the shells. Both figures show flow patterns along the shells which are little different from each other due to different baffle configurations; a isolated compartment layer and recirculation flow were formed in each compartment of shells. In the case of $15 \% \mathrm{BC}$ (Fig.4(a)), the flow field was characterized by flow zones of layer and recirculation flow formed in every baffle compartment, i.e., a vortex region and a high velocity region in the main flow. The figure also shows that the pattern of the vortex generated in shell was different for each compartment.

On the other hand, Fig.4(b) shows that the flow in the $30 \%$ baffle cut configuration had a remarkably different pattern from that in the $15 \%$ baffle cut. The mainstream velocity was restricted by adjacent baffle and wall of the shell, which makes flow cross throughout the entire shell. Therefore, the flow field was characterized by every compartment flow region. It was noticed that
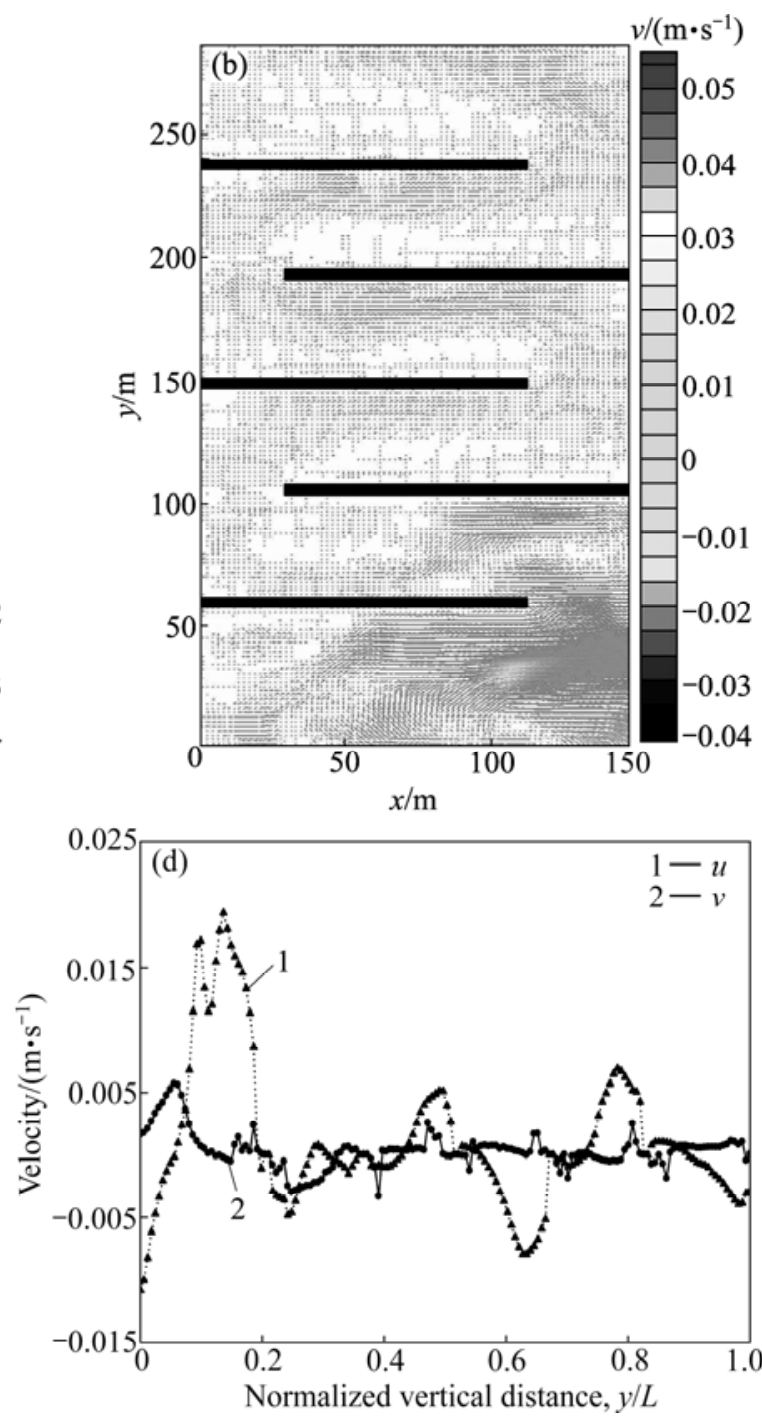
similar type of vortices in compartments except inlet and outlet was produced and a Karman vortex was not seen in any compartment of the entire shell. The reasons why this remarkable difference was noticed are the inlet flow condition and baffle cut. Figures 4(c) and 4(d) show the velocity distributions in the $y$ direction under the flow condition of $R e=4500$ in the inlet area for $15 \% \mathrm{BC}$ and $30 \%$ BC configurations, respectively. The $x$ and $y$ components of velocity, $u$ and $v$, were respectively normalized by $v_{0}$. In the $15 \% \mathrm{BC}$ configuration, the results show that $v$ had a maximum value in first compartment but in all other compartments, value of $v$ was noticed almost steady. The maximum value of $u$ was noticed in bottom and top compartment of the shell. Similarly, the third and top compartments were seen to have the minimum value of $u$ and this is due to the baffle configuration.

On the other hand, in the $30 \% \mathrm{BC}$ configuration, the result show that $v$ had a maximum fluctuation in bottom compartment but in all other compartments, the value of
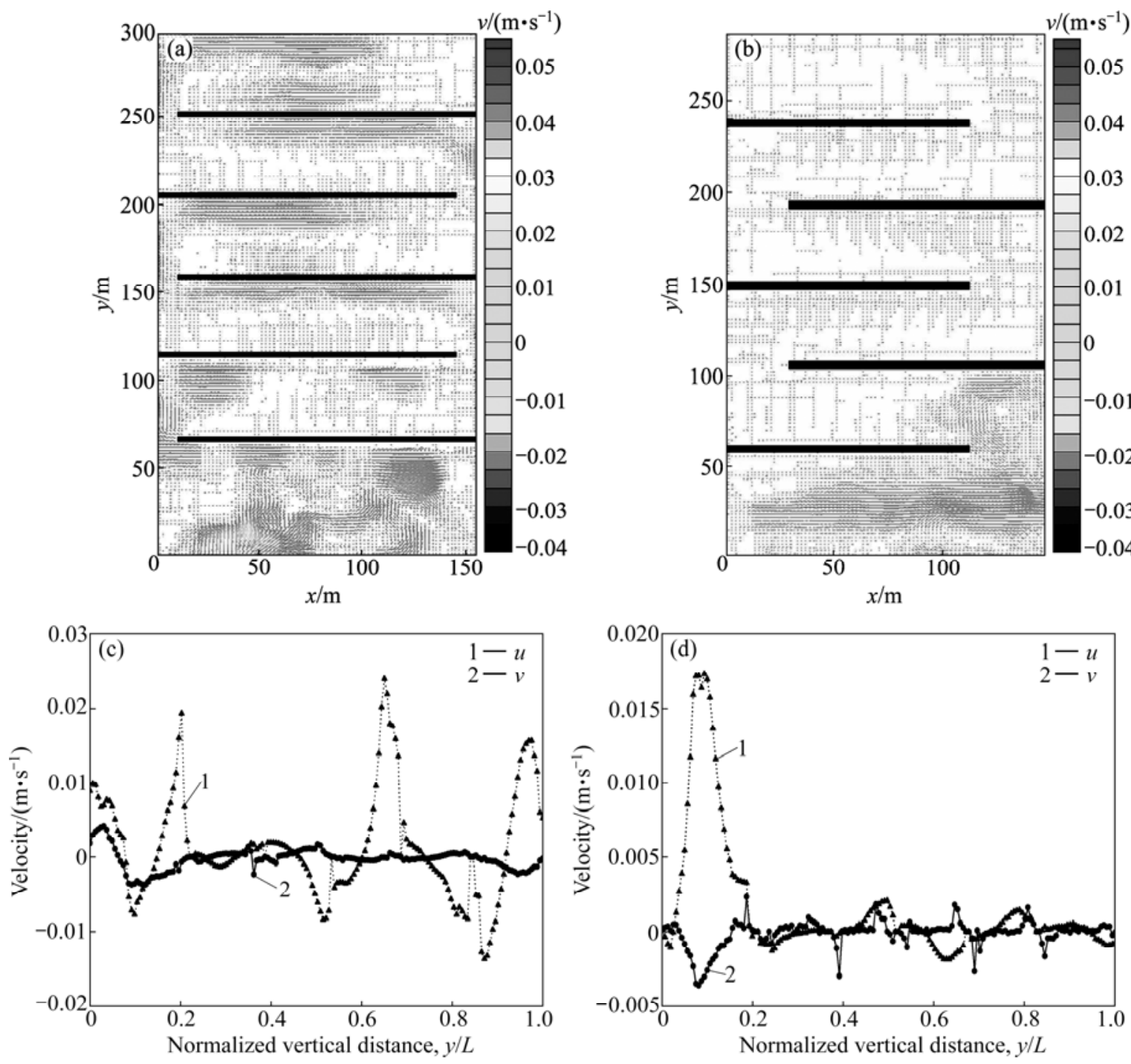

$v$ was noticed almost steady throughout the entire shell and this is because of the inlet flow direction. There was remarkable maximum value of $u$ in only bottom compartment of the shell. Similarly, $u$ was noticed to have the minimum value in the third and top compartments of the shell. These velocity distributions suggest that the flow mixing is more promoted in the $15 \% \mathrm{BC}$ than in the $30 \% \mathrm{BC}$ because of inlet flow direction and baffle cut.

\subsection{Velocity field and component distribution for $R e=$ 2500}

Figure 5 shows the results of the velocity vector field obtained for the $15 \% \mathrm{BC}$ and $30 \% \mathrm{BC}$ configurations in the whole flow areas of the test sections at the same $v_{0}$ $(=0.22 \mathrm{~m} / \mathrm{s})$ that also allows to have same $\operatorname{Re}(=2500)$. Figures 5(a) and 5(b) show the velocity vector in the shells. Both figures show flow pattern along the shells which are different from one another due to different baffle configurations; a separated compartment layer and

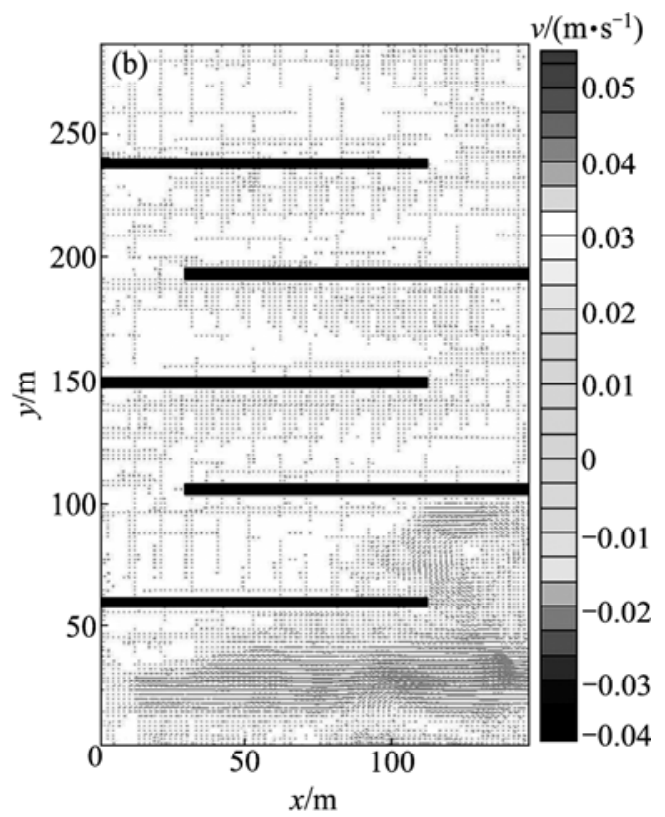

$v /\left(\mathrm{m} \cdot \mathrm{s}^{-1}\right)$ 
recirculation flow were formed in every compartment of shells. In the case of 15\% BC (Fig.5(a)), the flow field was featured by flow zones of every baffle compartment, i.e., a vortex region and a high velocity region in the main flow.

On the other hand, Fig.5(b) shows that the flow in the $30 \%$ baffle cut configuration had a remarkably different pattern from that in the $15 \%$ baffle cut. Here, the flow field was featured by flow region of every compartment. It was seen that similar type of vortices in compartments except inlet and outlet were produced [13-14]. The reasons why this remarkable difference was observed are the inlet flow condition and baffle cut. Figures 5(c) and 5(d) show the velocity distributions in the $y$ direction under the flow condition of $R e=2500$ in the inlet area for $15 \% \mathrm{BC}$ and $30 \% \mathrm{BC}$ configurations respectively. The $x$ and $y$ components of velocity, $u$ and $v$, were respectively normalized by $v_{0}$. In the $15 \% \mathrm{BC}$ configuration, the results show that $v$ had a maximum value bottom compartment but in all other compartments, value of $v$ was noticed almost steady. The maximum value of $u$ was noticed in bottom and top compartment of the shell. Similarly, the third and top compartments were seen to have minimum value of $u$ and this is due to the baffle configuration

On the other hand, in the $30 \% \mathrm{BC}$ configuration, the results show that $v$ had a maximum fluctuation in bottom compartment but in all other compartments, the value of $v$ was noticed almost steady throughout the entire shell and it is because of the inlet flow direction. There was remarkable maximum value of $u$ in only bottom compartment of the shell. Similarly, $u$ was noticed to fluctuate toward negative value.

\section{Conclusions}

1) The maximum velocity field was obtained for $15 \% \mathrm{BC}$ configuration having three various Reynolds numbers than $30 \% \mathrm{BC}$ configuration, which is due to the baffle cut and inlet flow direction.

2) The velocity component distribution for all three Reynolds number and both configurations show that the maximum fluctuation is noticed in $15 \% \mathrm{BC}$ than $30 \%$ $\mathrm{BC}$ configuration. The reason for this strange feature is due to the baffle cut condition and inlet flow direction.

\section{References}

[1] LEE Y H, LEE S C, JEONG H M, CHUNG H S, Experimental study on the characteristics of longitudinal fin air heating vaporizers in different seasons [J]. Journal of Mechanical Science and Technology, 2008, 22: 981-990.

[2] AIBA S, TSUCHIDA H, OTA T. Heat transfer around tubes in in-line tube banks [J]. B JSME, 1982, 25: 919-926.

[3] IWAKI C, CHEONG K H, MONJI H, MATSUI G. PIV measurement of the vertical cross-flow structure over tube bundles [J]. Experiments in Fluids, 2004, 37: 350-363.

[4] ZDRAVKOVICH M M. Interstitial flow field and fluid forces [C]// Technology for the '90s. ASME, 1993: 595-658.

[5] LORISCH W. Determination of heat transfer coefficients by a diffusion method [J]. VDI Mitteilungen Forchungsarbeit, 1929, 323: 46-68.

[6] PENDENNIS-WALLIS R. Photographic study of fluid flow between banks of tubes [J]. Engineering (IMechE.), 1939, 147: 423-426.

[7] BRADSHAW P. The effect of wind tunnel screens on nominally two-dimensional boundary layers [J]. J Fluid Mech, 1965, 22: 679-687.

[8] ZDRAVKOVICH M M. Smoke observation of wake of tandemcylinders [J]. Aeronaut J, 1972, 76: 108-114.

[9] WEAVER D S, AVD-RABBO A. A flow visualization study of a square array of tubes in water cross flow [J]. J Fluid Eng-T ASME, 1985, 107: 354-363.

[10] SIMONIN O, BARCOUDA M. Measurements and prediction of turbulent flow entering a staggered tube bundle [C]// Proceedings of the 4th International Symposium on Applications of Laser Anemometry to Fluid Mechanics. Lisbon, Portugal, 1988: 143-150.

[11] BALABANI S, YIANNESKIS M. Velocity characteristics of the cross flow over tube bundles [C]// Proceedings of the 7 th International Symposium on Applications of Laser Anemometry to Fluid Mechanics. Lisbon, Portugal, 1994: 317-331.

[12] UMEDA S, YANG W-J. Interaction of von Karman vortices and intersecting main streams in staggered tube bundles [J]. Exp Fluids, 1999, 26: 389-396.

[13] AIBA S, TSUCHIDA H, OTA T. Heat transfer around tubes in staggered tube banks [J]. B JSME, 1982, 25: 527-533.

[14] JEONG H M, CHUNG H S. Fluid flow characteristics in the aquaculture tank for a breeding fish [J]. KSME International Journal, 1998, 18(12): 2265-2272, 2004.

(Edited by YANG Bing) 